\title{
Obesidade centrípeta e disfunções metabólicas: patogenia, mensuração e papel profilático do exercício físico
}

\section{Centripetal obesity and metabolics malfunctions: patogeny, measurement and prophylatic role of physical exercise}

\author{
José Luciano Tavares da Silva ${ }^{1}$; Dartagnan Pinto Guedes ${ }^{2}$; Décio Sabattini \\ Barbosa $^{3}$; Jair Aparecido de Oliveira ${ }^{4}$; Joana E. Ribeiro Pinto Guedes ${ }^{5}$
}

\begin{abstract}
Resumo
Apesar do excesso de gordura no corpo ser considerado como fator de risco independente para o advento de disfunções degenerativas, a concentração de gordura localizada na região do tronco e do abdome e, principalmente, aquela disposta na região intra-abdominal ou visceral é a que apresenta maior associação com distúrbios metabólicos. A concentração de gordura centrípeta constitui-se em nítido fator de risco para a chamada síndrome metabólica, também conhecida como síndrome plurimetabólica ou simplesmente "síndrome X". Tal síndrome caracteriza-se por uma gama de disfunções endócrinometabólicas, induzindo seu portador a queda acentuada da qualidade de vida, devido a morbi-mortalidade decorrente. A prevalência da obesidade visceral e possíveis conseqüências degenerativas aparentam ser resultantes principalmente de distúrbios genéticos e endócrinos, podendo o diagnóstico ser realizado por intermédio de estimativas mediante medidas antropométricas ou por métodos de diagnóstico por imagem. Dentre os meios utilizados para a prevenção e tratamento dos altos níveis de adiposidade centrípeta, balanço energético negativo mediante reeducação alimentar e exercício físico demonstram alta eficiência, podendo a gordura localizada no tronco e no abdome, e principalmente a gordura visceral serem mobilizadas preferencialmente como fonte energética ao se confrontar com a gordura localizada em outras regiões do corpo. No que diz respeito ao exercício físico, não se observa consenso com relação ao tipo, à intensidade, à freqüência e à duração ideais, apesar de os exercícios aeróbios demonstrarem ser os mais efetivos..
\end{abstract}

Palavras Chave: Obesidade Centrípeta, Disfunções Metabólicas, Exercício Físico.

\footnotetext{
${ }^{1}$ Doutorando em Fisiologia Endócrina (Dept ${ }^{\circ}$ de Fisiologia e Biofísica, Instituto de Ciências Biomédicas, Universidade de São Paulo)

2 Professor da Universidade Estadual de Londrina (Dept ${ }^{\circ}$ de Fundamentos da Educação Física-CEFD), Doutor em Biodinâmica do Movimento Humano pela USP-SP

3 Professor da Universidade Estadual de Londrina (Dept ${ }^{\circ}$ de Patologia Aplicada, Legislação e Deontologia-CCS), Doutor em Patologia pela UNESP - Botucatu-SP)

${ }^{4}$ Professor da Universidade Estadual de Londrina (Dept ${ }^{\circ}$ de Patologia Aplicada, Legislação e Deontologia-CCS), Mestrando em Análises Clínicas - Minter - USP-UEL.

5 Professora da Universidade Estadual de Londrina (Dept ${ }^{\circ}$ de Fundamentos da Educação Física-CEFD), Mestre em Crescimento e Desenvolvimento Motor pela UFSM, Santa Maria-RS.
} 
Silva, J. L. T. da et al.

\begin{abstract}
Although fat excess in the body is considered as an independent risk factor for the establishment of degenerative malfunctions, the concentration of fat in the subcutaneous region of the trunk and abdomen and, especially the one located in the intra-abdominal or visceral region, shows closer association with metabolic disturbances. The centripetal fat concentration consists of a clear risk factor for the metabolic syndrome, also known as plurimetabolic syndrome or simply "syndrome X". This syndrome is characterized by a range of endocrine-metabolic malfunctions leading its carrier to a high impairment in his/her life quality, due to a resulting morbid mortality. The prevalence of visceral obesity and its possible degenerative consequences seem to be mainly a result of endocrine and genetic disturbances, being the diagnosis carried out through estimates for anthropometric measures or with methods of diagnosis by means of images. Among the resources used for the prevention and treatment of high levels of centripetal adiposity, a negative energetic balance through alimentary reeducation and physical exercise shows a high effectiveness. As a consequence, the trunk, abdomen and visceral fat may be preferably mobilized as an energetic source when compared to the fat located in other regions of the body. As for the physical exercise, there is no agreement in relation to the ideal type, intensity, frequency and duration, although the aerobic exercises prove to be the most effective ones.
\end{abstract}

Key words: Centripetal Obesity, Metabolic Malfunctions, Physical Exercise.

\section{Introdução}

A obesidade ocupa posição de realce com relação à complexidade e dificuldade de entendimento das alterações no corpo, tanto por parte dos leigos, quanto dos cientistas que a estudam. Milhares de pessoas sofrem deste mal, tanto as que constituem classes mais favorecidas da população, quanto as mais humildes,. sendo um problema nutricional de alta prevalência (FISBERG, 1995).

As implicações para a saúde que têm como causa as alterações associadas a esta patologia encontram-se bem fundamentadas. Apesar da complexidade do entendimento destas alterações, pesquisadores consideram-nas como possível fator de risco independente para patologias degenerativas (HUBERT et al., 1983), sendo sua incidência suficiente para causar alarde nas autoridades da saúde. Além disso, a obesidade não pode ser considerada como fator de risco apenas em adultos, tendo-se em vista que a obesidade infantil per se pode contribuir na elevação do risco de morbidade subseqüente, mesmo que o excesso de gordura não persista na idade adulta (MUST et al., 1992).

Estudos epidemiológicos realizados há mais de quinze anos apontaram na população norte- ameri- cana e européia altos níveis de prevalência, e conseqüentemente altos níveis de disfunções associadas à obesidade (VAN ITALLIE, 1985; KLUTHE; SCHUBERT, 1985). Com relação à população norte-americana, em 1980, aproximadamente 34 milhões de adultos foram considerados obesos, com custo econômico de 11,3 bilhões de dólares para o tratamento do diabetes mellitus tipo 2, 22,2 bilhões de dólares para doenças cardiovasculares, 1,5 bilhão para a hipertensão, todas disfunções orgânicas relacionadas com a morbidade e mortalidade ocasionadas pelo estado obeso (COLDITZ, 1992).

Com relação à incidência do problema na população brasileira, pode-se citar o estudo de PICCINI (1996) realizado na cidade de Pelotas - RS, no qual, utilizando-se como indicador de obesidade o índice de massa corporal (valores acima de $30 \mathrm{Kg} / \mathrm{m}^{2}$ ), observou-se incidência do problema em $15,3 \%$ da população masculina e $23,2 \%$ na população feminina naquela cidade. Além disso, segundo o Mninistério da Saúde (1993), por meio de dados brasileiros relacionados à prevalência de diabetes mellitus, observa-se que esta responde por $16 \%$ de óbitos por grupo de 100 mil habitantes para ambos os sexos. A variante do tipo 2 ou insulino-resistente desta patologia associa-se fortemente à obesidade, principalmente 
à obesidade localizada na região intra-abdominal ou visceral (BJÖRNTORP, 1985; LÖNNROTH, 1988), que, por sua vez, também se relaciona com doenças coronarianas (McKEIGUE; MARMOT, 1988).

Por conseguinte, observa-se a importância que a obesidade assume tanto relacionada com sua prevalência, quanto com disfunções subseqüentes. Todavia, é atualmente demonstrado que não apenas a deposição total de gordura no corpo, mas também a distribuição da gordura exerce importante influência nos níveis de risco proporcionados pela obesidade. Salienta-se, portanto, a existência de tipos e importâncias distintas com relação à obesidade no que tange à sua localização anatômica.

Mediante classificação proposta por Mcardle, Katch e Katch (1994), a obesidade pode apresentar-se como do: (a) tipo 1: excesso de gordura localizada proporcionalmente em todo o corpo (mais comum); (b) tipo 2: excesso de gordura localizada essencialmente na região subcutânea do tronco (andróide); tipo 3: excesso de gordura localizada essencialmente na região subcutânea da parte inferior do corpo (ginóide); e (d) tipo 4: excesso de gordura localizada essencialmente na região intra-abdominal (gordura visceral). Atenta-se que o acúmulo excessivo de gordura localizado na região subcutânea do tronco e abdome (tipo 2 ou andróide) e, principalmente, aquele localizado na região intra-abdominal ou visceral (tipo 4), ambas constituindo a chamada "obesidade centrípeta", demonstram as maiores associações com distúrbios metabólicos, contribuindo nitidamente para o advento de um estado mórbido, caracterizado por um conjunto de sinais e sintomas acompanhados por uma gama de disfunções endócrino-metabólicas, que conduzem seu portador à queda acentuada de qualidade de vida, além da possível mortalidade decorrente (síndrome metabólica) (DEFRONZO; FERRANNINI, 1991; KISSEBAH, 1997; BJÖRNTORP, 1997).

Desta maneira, observa-se que, apesar de o acúmulo excessivo de gordura corporal correlacionar-se com disfunções da saúde, este apre- senta malignidade menor ao se comparar com a obesidade de predominância central (YOUNG ; GELSKEY, 1995). Mesmo em se tratando de sujeitos geneticamente idênticos e discordantes no que diz respeito à massa de gordura corporal por questões ambientais, aparentemente só os que diferem quanto aos níveis de adiposidade localizada na região visceral caracterizam-se por maiores alterações na sensibilidade à insulina e intolerância à glicose (RÖNNEMAA et al., 1997). Neste aspecto , Yamashita et al. (1996) sugeriram o termo "síndrome da obesidade visceral" como um aglomerado de fatores de risco de forma independente do peso corporal e comparada a uma cascata de disfunções, tendo a aterosclerose como produto final.

Diante do exposto, a presente revisão tem por objetivo analisar as implicações da obesidade centrípeta em distúrbios metabólicos, além dos recursos utilizados para sua mensuração e o papel do exercício físico em sua profilaxia e tratamento.

\section{Disfunções associadas à obesidade centrípeta}

A gordura é um constituinte do corpo humano e, dentre outras, executa a importante função de reserva energética para mobilização em resposta às demandas metabólicas. A obesidade nada mais é que uma patologia caracterizada pelo excesso de gordura corporal, freqüentemente resultando em prejuízos à saúde.

Contudo, os depósitos de gordura não se constituem de maneira uniforme por todo o corpo. As células de gordura localizadas ao redor da cintura e flancos e no abdome são mais ativas metabolicamente que aquelas localizadas nas coxas e nádegas (NATIONAL INSTITUTES OF HEALTH CONSENSUS DEVELOPMENT PANEL ON THE HEALTH IMPLICATIONS OF OBESITY, 1985). Ao revisar estudos concernentes ao papel da gordura corporal no desenvolvimento da resistência à insulina na obesidade, Lönnroth (1988) inferiu que a alta taxa de lipólise associada à obesidade abdomi- 
nal poderia conduzir à resistência periférica à insulina, sendo tal hipótese suportada por diversos estudos experimentais realizados nesta área (DESPRÉS et al., 1989; HENNES; SHRAGO; KISSSEBAH, 1990, CAREY et al., 1996; MARCOR et al., 1997; RÖNNEMAA et al., 1997).

Os estudos de VAGUE (1947; 1956) diferenciaram os tipos de obesidade no que tange à sua localização anatômica (andróide e ginóide) e verificaram que o padrão andróide associava-se relevantemente aos fatores que predispunham ao risco de ser obeso (aterosclerose, hiperuricemia e diabetes mellitus). A partir desses estudos, mais recentemente outras importantes pesquisas foram realizadas (KISSEBAH, 1982; REAVEN, 1988; KAPLAN, 1989; BJÖRNTORP, 1990, FUJIMOTO et al., 1995; YAMASHITA et al., 1996; KURIYAMA et al., 1998; SILVA, 1999), confirmando a relevância do estudo da distribuição da gordura no corpo e os fatores de risco a ela associados. Segundo Kissebah (1997), a concentração excessiva de gordura na região abdominal é considerada fator de risco associado com morbidades e mortalidade elevadas, desempenhando papel causal em um aglomerado de anormalidades metabólicas e caracterizando uma síndrome, incluindo-se como componentes clínicos a intolerância à glicose, o diabetes mellitus tipo 2, a dislipidemia, a hipertensão e a aterosclerose. Esta síndrome metabólica, também conhecida por "síndrome X ", pode ter seu início já na infância, com a quantidade de gordura correlacionando-se com a sensibilidade à insulina mesmo em crianças não-obesas, contribuindo conseqüentemente para o risco de disfunções degenerativas na vida adulta (ARSLANIAN ; SUPRASONGSIN, 1996).

Por intermédio de pesquisas conduzidas com o objetivo de observar associações entre o padrão de distribuição de gordura corporal e disfunções orgânicas, verificou-se que mudanças hormonais aliadas à predisposição genética poderiam conduzir à maior concentração de gordura abdominal (BJÖRNTORP, 1995a). As mudanças hormonais são devidas à estimulação do eixo hipotalâmico-hipofisário-adrenal (eixo HPA) que por sua vez conduzirão à anormalidades endócrinas, como distúrbios na secreção de cortisol, hormônio do crescimento (GH) e esteróides sexuais. Estas alterações hormonais poderão aumentar a deposição de gordura visceral, que, juntamente com distúrbios neuroendócrinos, excesso de ingestão calórica e inatividade física, poderão todos estar envolvidos no desenvolvimento de resistência à insulina, doenças cardiovasculares e diabetes mellitus tipo 2 (BJÖRNTORP, 1995a).

Uma característica peculiar da gordura visceral é sua elevada atividade lipogênica, tanto quanto sua acelerada atividade lipolítica, elevando portanto os níveis de ácidos graxos livres na circulação. Níveis elevados de AGL no sangue portal poderão eventualmente resultar em elevação na síntese de triacilgliceróis hepáticos, causando hiperlipidemia, além de induzir à resistência à insulina, intolerância à glicose, hipertensão e finalmente aterosclerose (YAMASHITA et al., 1996).

Para Benthem et al.(2000), a elevação na quantidade de ácidos graxos de cadeia longa drenados em direção ao fígado por intermédio da veia porta, pode conduzir à ativação do eixo HPA e sistema simpático, com concomitante desenvolvimento de pressão arterial média elevada e resistência à insulina.

Não obstante, Kissebah (1997) observou que aproximadamente $80 \%$ dos indivíduos portadores de excesso de gordura na região intra-abdominal ou visceral são também obesos, muitos apresentando diabetes mellitus tipo 2 ou tolerância à glicose diminuída. A obesidade pode precipitar o início do diabetes ou agravar patologias já existentes, ao passo que a perda de peso corporal melhora ou até mesmo reverte as anormalidades associadas à glicose e à homeostase da insulina. No entanto, a prevalência de diabetes em mulheres com predominância de gordura nas regiões superiores do corpo (padrão andróide) é três vezes maior que em mulheres com padrão de gordura nas regiões inferiores do corpo (padrão ginóide): as primeiras possuem risco relati- 
vo de apresentar diabetes 10,3 vezes maior que as segundas. Portanto, observa-se que os efeitos da obesidade e distribuição de gordura corporal na prevalência do diabetes são independentes um do outro, porém aditivos.

Em estudo relativo à adiposidade visceral, insulina plasmática em jejum e pressão sangüínea em descendentes de japoneses, Bokyo et al. (1995) verificaram a existência de papel, possivelmente casual da insulina (ou resistência à insulina) no desenvolvimento de altos valores de pressão arterial. Os dados deste estudo também sugeriram que a adiposidade visceral pode afetar a pressão arterial por intermédio de mecanismos mediados ou não pela insulina. Essa sugestão pode ainda ser reforçada pelo estudo de Nakajima et al. (1989), com relação à presença de associação entre o desempenho do ventrículo esquerdo e o acúmulo de gordura intra-abdominal em indivíduos obesos subdivididos em dois grupos de acordo com o acúmulo de gordura visceral. Os autores observaram elevação significante no índice de dimensão diastólica e volume sistólico nos sujeitos que apresentaram acúmulo excessivo de gordura visceral, refletindo presença de um estado hipervolêmico nestes sujeitos.

Mesmo em se tratando da gordura subcutânea, porém localizada na região abdominal nota-se também distinções em comparação com a gordura subcutânea localizada em outras regiões do organismo. Além disso, em se tratando apenas da gordura subcutânea abdominal, os adipócitos situados mais profundamente também apresentam distinções daqueles mais superficiais. Kelley et al.(2000) observaram que o tecido subcutâneo abdominal profundo relaciona-se fortemente com a resistência à insulina de uma maneira quase idêntica ao tecido adiposo visceral. Por outro lado, o tecido adiposo subcutâneo abdominal superficial apresenta fraca associação com a sensibilidade insulínica, próximo ao padrão observado no tecido adiposo subcutâneo glúteofemoral.

\section{Fatores associados à obesidade centrípeta}

\section{a) Fatores genéticos}

O motivo que leva indivíduos obesos a apresentarem um ou outro padrão de distribuição de gordura ainda não se apresenta claro. Contudo, observa-se que a hereditariedade desempenha importante papel na distribuição centrípeta da gordura (BOUCHARD et al., 1990). O mecanismo associado a este efeito genético parece ser bastante heterogêneo (BOUCHARD, 1988). De acordo com Samáras et al. (1997), os antecedentes genéticos aparentam contribuir expressivamente para o advento da adiposidade total e centrípeta, sugerindo-se que alguns dos genes responsáveis pela adiposidade centrípeta e suas sequielas metabólicas seriam diferentes daqueles responsáveis pela adiposidade total.

Corroborando tais achados, de acordo com Mauriège e Bouchard (1996), o fenótipo obesidade pode ser determinado parcialmente por diversos genes ou mesmo por um único gene principal, sendo um dos candidatos o receptor $\mathrm{b}_{3}$ adrenérgico. Este é o principal receptor que medeia a termogênese estimulada pelas catecolaminas no tecido adiposo marrom, o qual, no ser humano, encontra-se espalhado nos grandes vasos do tórax e abdome, diferindo-se do tecido adiposo branco no que diz respeito ao seu maior número de mitocôndrias e possuindo a função de oxidação de lipídios para produzir calor e remover a gordura em excesso. $\mathrm{O}$ tecido adiposo branco (incluindo o tecido adiposo subcutâneo e o visceral) é muito mais abundante, servindo como reserva de gordura, a qual poderá ser mobilizada por lipólise para a geração de AGL, que por sua vez é utilizado por outros tecidos.

De acordo com Newsholme e Leech (1985), taxas controladas de termogênese no tecido adiposo marrom poderiam ser importantes em "queimar" o excesso de energia consumida na dieta, levando à manutenção do peso corporal. Sujeitos obesos poderiam, portanto, sofrer de um "mal funcionamento" 
do tecido adiposo marrom. Embora o tecido adiposo marrom não seja proeminente no ser humano, demonstrou-se recentemente sua atividade em indivíduos normais, exercendo sua função na "termogênese induzida pela dieta". Tal fato poderia de certa forma, explicar o porquê de algumas pessoas "comerem sem engordar". Verificou-se que este tecido é insignificante ou não existe em obesos (MAYES, 1998).

$\mathrm{O}$ receptor $\mathrm{b}_{3}$ adrenérgico também é importante para mediar a estimulação da lipólise pelas catecolaminas nas células adiposas brancas de diversas espécies animais, incluindo o ser humano (ARNER, 1995). Segundo Hoffstedt (1996), a função do receptor $b_{3}$ adrenérgico encontra-se fortemente relacionada com a obesidade visceral e seus vários distúrbios metabólicos. Uma mutação no códon 64 do gen deste receptor, resultando na substituição do aminoácido triptofano pela arginina na primeira alça intracelular da proteína, vem sendo estudada em diversas populações (WALSTON et al., 1995; WÍDEN et al., 1995; CLÉMENT et al., 1995). Considera-se que tal mutação estaria envolvida no início do diabetes mellitus tipo 2 (WALSTON et al., 1995; WÍDEN et al., 1995), na resistência à insulina (WÍDEN et al., 1995), e, do mesmo modo, no ganho de peso em sujeitos morbidamente obesos (CLÉMENT et al., 1995).

Apesar de esses achados serem contestados pelos estudos apresentados por Elbein et al. (1996) e Nagase et al. (1997), os quais não encontraram tais relações, para Giacobino (1995) a atividade teoricamente baixa atividade de receptores $b_{3}$ adrenérgicos pode promover a obesidade de diversas maneiras. Delas, a mais importante se dá provavelmente mediante diminuição da termogênese no tecido adiposo marrom, o qual, de acordo com Lowell e Flier (1995), aparentemente é o responsável pela intermediação entre a atividade destes receptores e a elevação do dispêndio energético.

Além disso, a função diminuída do receptor no tecido adiposo branco poderia diminuir a lipólise e, através disso, causar a retenção de lipídios nas célu- las de gordura. Em humanos, esta última pode ser contribuidor especialmente importante para a obesidade visceral. Segundo Widén et al. (1995) pode-se ainda considerar o receptor $b_{3}$ adrenérgico agindo como elo de ligação entre a obesidade centrípeta e a resistência à insulina. De acordo com Silver et al. (1996), a mutação genética citada associa-se a características da Síndrome X, incluindo além da obesidade centrípeta, a hiperinsulinemia/resistência à insulina, elevação da pressão arterial diastólica, além de aparecimento precoce do diabetes mellitus tipo 2 .

Tal ligação pode ser explicada por intermédio de uma sobrefunção destes receptores, uma vez instalada a obesidade visceral. Uma elevação em sua sensibilidade lipolítica em resposta às catecolaminas pode conduzir a um aumento na liberação de ácidos graxos não esterificados para dentro da circulação portal, com efeitos adversos para a função hepática, incluindo prejuízo no metabolismo da glicose, lipoproteínas e insulina, conduzindo à síndrome metabólica (HOFFSTEDT, 1996).

Além dos receptores adrenérgicos, receptores para glicocorticóides pouco funcionais devido a alterações genéticas podem também acentuar os efeitos adversos à saúde provocados pela excessiva concentração sangüínea de cortisol, como observado na Síndrome de Cushing que tem como fator etiológico a hipercortisolemia, cujos sinais e sintomas assemelham-se à Síndrome Metabólica (ROSMOND; BOUCHARD; BJÖRNTORP, 2001).

\section{b) Fatores endócrinos}

As alterações endócrinas múltiplas associadas à obesidade abdominal podem ser tanto responsáveis primárias, como conseqüência da condição de obesidade (JOHANNSSON et al., 1997). As características do tecido adiposo demonstram que indivíduos do sexo feminino possuem localização deste tecido mais predominantemente na região subcutânea glúteo-femoral, ao passo que o sexo masculino apresenta grande proporção de adipócitos na região cen- 
tral (intra-abdominal e subcutânea). A obesidade glúteo-femoral, uma particularidade do sexo feminino, caracteriza-se por aumento na massa de gordura corporal, distribuída em um padrão fisiologicamente esperado na mulher. Aparentemente, pode-se considerar o advento de uma anormalidade endócrina quando o acúmulo de gordura corporal no sexo feminino segue um padrão masculino, com predominância de tecido adiposo centrípeto (BJÖRNTORP, 1997).

Em se tratando do sexo masculino, apesar de os homens possuírem proporção maior de gordura em depósitos centrais, quando tais depósitos são exagerados, as anomalias endócrinas parecem ser mais prevalentes, observando-se ser este exagero o ponto chave destas anormalidades na obesidade (BJÖRNTORP, 1997). A distribuição de gordura corporal e as anormalidades metabólicas que a acompanham podem, portanto, ser manifestação de desordem no equilíbrio androgênico/estrogênico e desenvolvimento de dismorfismo sexual. No entanto, segundo Björntorp (1996), se no sexo masculino os valores de testosterona permanecem dentro de uma "janela" de classe normal, observa-se sensibilidade à insulina ótima, o mesmo acontecendo no sexo feminino. Não obstante, a falta de estrogênios é, provavelmente um fator mais importante que o relativamente pequeno excesso de androgênios na mulher hiperandrogênica para o acúmulo de gordura visceral. Além disso, outros hormônios como o cortisol e o hormônio do crescimento $(\mathrm{GH})$ provavelmente também estão envolvidos, sobrepujando os efeitos dos hormônios androgênicos (BJÖRNTORP, 1997).

Com relação ao cortisol, este parece influenciar a distribuição regional de gordura (KISSEBAH, 1997), observando-se que a secreção urinária de cortisol livre é diretamente proporcional à relação entre circunferência da cintura e do quadril, e particularmente com a circunferência da cintura, indicadores de depósitos de gordura localizados na região central do corpo. Sugere-se que mulheres que apresentam acúmulo de gordura na região visceral demonstram sensibilidade elevada no eixo hipotalâmicohipofisário-adrenal, causando maior deposição de gordura central (MARIN, et al., 1992). Outrossim, segundo Pasquali et al. (1993) mulheres obesas que apresentam distribuição de gordura predominantemente abdominal podem apresentar hiperatividade do eixo hipotalâmico-hipofisário-adrenal, representando fenômeno adaptativo secundário a estado de resistência funcional ao cortisol.

De acordo com Björntorp (1991) a gordura visceral é um tecido-alvo para a atividade dos glicocorticóides devido a elevado número de receptores (intra-celulares) para este hormônio, o qual eleva a atividade das enzimas-chaves para a lipogênese neste tecido.

Uma enzima relacionada com a regeneração de glicocorticóides ativos a partir de inativos, a 11 beta hidroxiesteróide desidrogenase-1 (11 beta HSD-1) aparentemente se encontra envolvida na etiologia da obesidade visceral. Quando super-expressada no tecido adiposo, esta enzima demonstrou promover níveis elevados de adipócitos viscerais. A obesidade visceral resultante associa-se com resistência à insulina e dislipidemia (WOLF et al., 2002). Camundongos transgênicos que super-expressam a 11 beta HSD-1 seletivamente no tecido adiposo (numa extensão similar à encontrada no tecido adiposo de pessoas obesas) apresentam níveis elevados de corticosterona no tecido adiposo, desenvolvendo obesidade visceral. Os animais também desenvolveram resistência insulínica pronunciada, hiperlipidemia, hiperfagia e hiperleptinemia. A atividade aumentada da 11 beta HSD-1 nos adipócitos pode ser, portanto, a etiologia molecular comum para a obesidade visceral e síndrome metabólica (MASUZAKI et al., 2001).

Ao contrário do cortisol, no que diz respeito ao $\mathrm{GH}$, sugere-se que secreção diminuída (e não aumentada) desse hormônio pode ser importante fator para o desenvolvimento de conseqüências metabólicas e circulatórias na obesidade visceral. Com efeito, ao se administrar $\mathrm{GH}$ em indivíduos que apresentam alta deposição de gordura visceral, comprovouse efeito favorável para a redução destes depósitos, além de se verificar o aumento na sensibilidade à 
insulina e efeitos favoráveis no metabolismo das lipoproteínas e pressão arterial diastólica (JOHANNSSON et al., 1997). Logo, os níveis plasmáticos e o pulso de secreção de GH são baixos na obesidade, estando este hormônio claramente envolvido na regulação da massa de gordura visceral em humanos. Os mecanismos celulares atrás destas mudanças têm sido parcialmente revelados, com a maioria destas interações parecendo requerer um sinergismo com os hormônios esteróides (androgênios, estrogênios, cortisol, etc) (BJÖRNTORP, 1997).

\section{Recursos utilizados para a mensuração da gor- dura centrípeta}

Em virtude da distribuição de gordura corporal predizer, de forma independente, as aberrações metabólicas que conduzem às disfunções apresentadas, os meios para quantificá-la adquirem considerável importância (KISSEBAH, 1997).

\section{a) Avaliação por métodos antropométricos}

A técnica de estimativa da distribuição da gordura corporal por intermédio das espessuras das dobras cutâneas demonstra algumas relações com parâmetros metabólicos, porém com carência de força de predição. No entanto, medidas de circunferência de cintura e a relação entre as medidas de circunferência da cintura de quadril são utilizadas constantemente para estimar a distribuição de gordura corporal, apresentando por vezes altas correlações com resultados obtidos mediante métodos de mensuração mais fidedignos.

Esforços para padronização de medidas de circunferências de cintura e quadril têm eliminado consideravelmente variações neste procedimento, exceto no caso de obesidade mórbida. Jakicic et al. (1993), verificou os pontos que melhor se correlacionam com parâmetros de lipídeos e lipoproteínas séricos, ou seja, o ponto médio entre a última costela e a crista ilíaca para a cintura e a maior circunferência de glúteos para o quadril.
De acordo com Björntorp (1985), o risco de disfunções eleva-se abruptamente quando a relação entre a circunferência da cintura e do quadril elevase acima de 1,0 para homens e 0,8 para mulheres, ou, de acordo com Mcardle. Katch e Katch (1994), quando se eleva acima de 0,8 para mulheres e 0,95 para homens. Segundo KISSEBAH (1988), aumento na relação entre circunferência da cintura e do quadril é acompanhado por elevação progressiva da glicose e insulina plasmáticas em jejum, além de altas respostas ao teste oral de tolerância à glicose, demonstrando uma associação com obesidade centrípeta e conseqüentes disfunções metabólicas.

Porém, aparentemente, a circunferência de cintura é uma medida superior à relação cintura/quadril para a predição de anormalidades metabólicas, observando-se que circunferência de cintura de cerca de $100 \mathrm{~cm}$ pode representar limiar acima do qual as complicações metabólicas são mais suscetíveis de serem encontradas (POULIOT et al., 1994). Colman et al. (1995), concluíram que juntamente com a gordura corporal total, a circunferência da cintura mostrou-se um indicador independente dos níveis de insulina em jejum em homens e mulheres saudáveis. Por sua vez, Branchtein et al. (1997), ao estudarem distribuição de gordura centrípeta no decorrer da gravidez como indicador da intolerância à glicose no período gestacional, concluíram que a distribuição de gordura medida pela relação entre a circunferência da cintura e do quadril, ou pela circunferência da cintura associou-se de forma independente com a tolerância à glicose no período de gestação.

Não obstante, apesar de sua incontestável praticidade e baixo custo, a utilização da antropometria para estimação da obesidade visceral é sujeita a erros significantes quando pouca atenção é dispensada ao treinamento de avaliadores (KISSEBAH, 1997). Além disso, por tais métodos não se observa separação entre os depósitos de gordura subcutânea e visceral. Dessa maneira, a avaliação da obesidade centrípeta mediante métodos de imagem poderá proporcionar mensurações mais fidedignas e menos sujeitas a erros de estimativa, prin- 
cipalmente por intermédio de técnicas que permitam diferenciação entre os dois depósitos.

\section{b) Avaliação por métodos de imagem}

Després et al. (1990) observaram diversas limitações na utilização de métodos antropométricos como única medida da distribuição de gordura em pesquisas científicas, defendendo a inclusão da tomografia computadorizada (TC) ou ressonância nuclear magnética (RNM) para obtenção de informações quanto à gordura visceral, separadamente daquela localizada na região subcutânea.

$\mathrm{Na} \mathrm{TC}$, os raios X passam através do tecido, atenuando-se como resultado da dispersão, absorção fotoelétrica e outras interações (HEYMSFIELD; NOEL apud KISSEBAH, 1997). As diferentes atenuações produzidas pelo tecido magro permitem separações visuais e matemáticas dos componentes da imagem, com o software utilizado delineando áreas teciduais para estimativas das quantidades de tecido adiposo, músculo ou osso (KISSEBAH, 1997).

Peiris et al. (1988) relataram que a avaliação da distribuição da gordura no interior do abdome mediante medição da área de gordura total, intra-abdominal e subcutânea em três partes contíguas com o centro localizado no plano médio da $4^{\text {a }}$ vértebra lombar é o suficiente para a definição de fortes correlações com parâmetros metabólicos. Nesse sentido, de acordo com Busetto et al. (1992), a TC é considerada o método mais confiável para a mensuração de depósitos de gordura tanto visceral quanto subcutânea. Para Kissebah (1997), a medição da distribuição de gordura por TC pode ser o melhor indicador global de fatores de risco metabólicos e cardiovasculares, sendo extremamente reprodutiva e possuindo maior valor preditivo. Contudo, a utilização em larga escala da TC não é viável em virtude da exposição do avaliado à alta radiação ionizante, alto preço e baixa disponibilidade.

Um segundo método de imagem para a mensuração da gordura visceral é a imagem por res- sonância nuclear magnética (RNM), cuja utilização é bastante segura em gestantes e crianças, devido ao fato de não utilizar radiação ionizante (KISSEBAH, 1997). Na RNM, a radiação eletromagnética, na presença de um forte campo magnético, é utilizada para a excitação do núcleo de hidrogênio das moléculas de água e gordura, levando à emissão de um sinal detectável, o qual pode ser então reajustado, sob o controle do computador, para proporcionar uma representação visual dos tecidos corporais (McARDLE ; KATCH; KATCH, 1994).

Para Després et al. (1996), comparações realizadas com a TC sugerem que a RNM proporcionam imagens do abdome com detalhes anatômicos similares, esperando-se contudo um erro aproximado de $10 \%$ na quantidade de gordura visceral. Todavia, da mesma maneira que a TC, sua utilização é limitada pelo seu alto custo, pela dificuldade de acesso e pelo tempo dispendido em sua utilização (KISSEBAH, 1997).

O terceiro método de imagem utilizado para a estimativa da gordura visceral é a absorciometria de raios $X$ de dupla energia (DEXA), a qual tem permitido medidas rápidas e não invasivas de tecido ósseo e estimativa de composição corporal, com mínimo de exposição à radiação (CLASEY et al., 1997). Apesar de ser proposta para a mensuração do conteúdo mineral ósseo, o DEXA pode ser utilizado para a estimativa dos tecidos corporais moles (KOHRT, 1995), envolvendo radiações desprezíveis (HANSEN et al., 1993) e oferecendo custos mais razoáveis que a TC ou a RNM. Através da melhoria da qualidade do software desta técnica observam-se elevações em sua precisão e exatidão (CLASEY et al., 1997).

O princípio da absorciometria baseia-se na atenuação exponencial dos raios $\mathrm{X}$ por intermédio de duas energias quando estes passam através dos tecidos corporais. A absorciometria de fóton duplo (DPA), que vem sendo utilizada por mais de 15 anos, utiliza fonte radionucleica, usualmente $\mathrm{o}^{153} \mathrm{Gd}$, para a geração de raios gama. Nas máquinas DEXA modernas, a fonte radionucleica é colocada em um tubo com baixa corrente de raios-X, oferecendo au- 
mento de resolução, precisão, tempo de exame reduzido e menor manutenção (JEBB et al., 1997).

No entanto, observa-se que, apesar de poder estimar a quantidade de gordura visceral, a utilização da DEXA poderá apresentar certas limitações dependendo da população avaliada. Segundo Clasey et al.(1997), para estimativa da quantidade de gordura em idosos o DEXA resulta em erros variáveis, tornando-o um método inaceitável para a utilização única em pesquisas de composição corporal nesta população. Portanto, mesmo demonstrado ser mais um método para a mensuração da composição corporal, permanecem dúvidas sobre a exatidão destas medidas. Os fatores que contribuem para a incerteza com relação à validade deste equipamento, são a variabilidade existente entre os fabricantes no que diz respeito aos métodos de calibração, aquisição e análise de dados (KOHRT, 1995).

\section{Papel do exercício físico na prevenção e no tra- tamento da obesidade centrípeta}

Há várias modalidades de tratamento da obesidade, entre as quais se incluem as técnicas designadas a modificações na ingestão calórica, a melhora do conhecimento acerca da nutrição e a elevação no nível de prática de atividade física, além das opções cirúrgicas e farmacológicas. Contudo, a simples modificação do comportamento, tanto alimentar como relacionado ao nível de atividade física, tem sido proposta como uma das mais efetivas técnicas, tanto para sua prevenção quanto para seu tratamento (WILMORE; COSTILL, 1994).

Considerando apenas uma questão matemática, o peso corporal é diminuído quando o dispêndio calórico supera sua ingestão, sendo a recíproca verdadeira, ou seja, o peso corporal é aumentado quando a ingestão supera o dispêndio energético. Todavia, apesar de mudanças no balanço energético serem acompanhadas por mudanças no peso corporal, a natureza da mudança do peso corporal varia acentuadamente de acordo com os comportamentos específicos que conduzem ao desequilíbrio energético.
Por exemplo, jejum e restrição calórica extrema causam perdas substanciais de água e massa corporal magra (massa muscular e todo tecido corporal que não é gordura), ao passo que balanço calórico negativo induzido pelo exercício físico resulta em perda de peso corporal que consista essencialmente de gordura (AMERICAN COLLEGE OF SPORTS MEDICINE, 1995), desde que, obviamente, seguindo-se dietas adequadas ao dispêndio energético pretendido, evitando-se perdas de outros tecidos.

Especificamente para a adiposidade centrípeta, a restrição energética por intermédio de dietas hipocalóricas tem sido o principal meio para a perda de gordura, principalmente a localizada na região visceral. Apesar de esta perda ser diretamente proporcional à redução do peso corporal, observa-se uma mobilização preferencial da gordura intra-abdominal em comparação com a subcutânea, demonstrando que os adipócitos situados na região mesentérica são mais sensíveis à estimulação lipolítica da dieta que aqueles situados na região subcutânea abdominal e femoral, porém advertindo-se para grande variação individual (ROSS, 1997). De acordo com Busetto (2001) redução rápida e preferencial na massa adiposa visceral ocorre no decorrer da primeira fase da perda de peso em pacientes morbidamente obesos, possivelmente como resultado de uma ativação do Sistema Nervoso Autônomo Simpático.

Para Björntorp (1995b), o exercício físico e a conseqüente redução do peso corporal já provaram bastante eficiência no tratamento da obesidade centrípeta e resistência à insulina. Quando exercícios aeróbios são utilizados para induzir a perda de peso corporal, os homens geralmente perdem mais gordura que as mulheres, observando-se que no sexo masculino a redução de gordura apresenta predominância centrípeta, reduzindo potencialmente os riscos de doenças cardiovasculares. Relativa resistência à perda de peso corporal pode ser observada nas mulheres quando comparadas com os homens. Contudo, apesar desta resistência à perda de peso corporal notada nas mulheres, aquelas com distribuição 
de gordura típica do sexo masculino são fortemente beneficiadas pelo exercício aeróbio (DESPRÉS et al., 1988).

Estes mesmos autores observaram ainda a existência de mobilização preferencial da gordura subcutânea do tronco quando comparada com aquela localizada na região periférica apesar de não terem confirmado se havia a existência de mobilização preferencial também da gordura visceral ou intra-abdominal.

No que diz respeito ao compartimento visceral, Yamashita et al. (1996) citam que lutadores de sumô (esporte nacional no Japão), os quais se submetem a altos índices de exercício físico intenso diário, também apresentam elevadas taxas de gordura subcutânea total, haja vista a alta ingestão calórica (acima de $7000 \mathrm{Kcal} / \mathrm{dia}$ ). No entanto, tais lutadores demonstram acúmulo de gordura somente nesta região (juntamente com grande desenvolvimento muscular), sendo o acúmulo de gordura visceral muito ameno quando comparado com aquele em homens obesos com um índice de massa corporal similar. Observouse ainda que a maioria dos lutadores de sumô analisados neste estudo apresentaram níveis de glicose e triacilgliceróis normais, além de baixos níveis de colesterol, apesar de marcada obesidade. Estes dados sugerem, segundo os autores, que o exercício físico pode prevenir o acúmulo de gordura visceral, diminuindo a lipogênese neste compartimento e, conseqüentemente, o advir de suas implicações deletérias à saúde, mesmo mantendo-se altos níveis de adiposidade total.

Em outro estudo, utilizando-se a técnica de TC para avaliar a gordura visceral, observou-se relação inversa entre atividade física e depósitos centrípetos de gordura (HUNTER et al., 1997). O exercício físico regular pode, portanto, ser um importante meio para a prevenção do ganho de peso corporal e o desenvolvimento da obesidade abdominal, podendo, dentre outros benefícios, aumentar a sensibilidade à insulina e alterar favoravelmente o perfil de lipoproteínas plasmáticas (BUEMANN; TREMBLAY, 1996).
Em indivíduos já acometidos por disfunções cujo fator de risco principal é a deposição de gordura centrípeta, o exercício físico também pode agir favoravelmente em sua recuperação. Tal suposição é fortalecida por Lehmann et al. (1995), em estudo relacionado à perda de gordura abdominal e à melhora no perfil de risco cardiovascular pela prática de exercício aeróbio moderado em indivíduos portadores de diabetes mellitus tipo 2. Esses autores observaram diminuição considerável na gordura corporal total, particularmente na região abdominal, medida pela relação cintura-quadril. Observaram ainda que a diminuição na gordura corporal não foi acompanhada pela perda de peso corporal total, dando a idéia de que pessoas com reservas elevadas de gordura são "protegidas" da perda de massa corporal magra, 'a medida que ocorre a perda de peso corporal. Neste estudo, a massa corporal magra aumentou, enquanto a massa de gordura, particularmente a gordura abdominal, diminuiu.

Os mecanismos mediados pelo exercício físico, responsáveis por estas mudanças metabólicas favoráveis, devem-se provavelmente a alterações na atividade do transportador de glicose sensível à insulina (GLUT4) e à elevação da atividade da enzima lipase lipoprotéica (LPL) na musculatura esquelética. A elevação da atividade da LPL muscular pode propiciar ação favorável na sensibilidade à insulina e no metabolismo das lipoproteínas (BUEMANN; TREMBLAY, 1996). Uma elevação na quantidade do transportador de glicose GLUT4 devido ao exercício aeróbio, pode prover os músculos de um mecanismo capaz de remover a glicose do sangue, elevando a disposição deste substrato energético para a contração muscular (DENGEL et al., 1996).

De fato, Houmard et al. (1995) demonstraram que o exercício físico em cicloergômetro, uma hora por dia a $76 \%$ do consumo máximo de oxigênio por apenas sete dias, proporcionou elevação de média de 2,8 vezes no transportador de glicose GLUT4, cuja quantidade relaciona-se com a melhora da sensibilidade à insulina de todo o organismo, exercendo portanto papel vital no metabolismo de carboidratos. 
Além da elevação quantitativa de GLUT4 na célula, aparentemente a sinalização insulínica em eventos pós-receptor também torna-se mais eficiente após condicionamento aeróbio. De acordo com Kirwan et al. (2000) o consumo máximo de oxigênio encontra-se fortemente correlacionado com a ativação da PI3-kinase na cascata de sinalização insulínica responsável pela translocação do GLUT4 do citosol para a membrana plasmática, aumentando, portanto, a captação de glicose pela célula.

Contudo, o impacto do exercício físico (tanto na sensibilidade à insulina quanto na atividade da LPL), aparenta ser de curta duração, e a manutenção de um programa de exercício físico regular é portanto recomendado como condição para que se possa alcançar os efeitos ótimos nos fatores de risco cardiovascular (BUEMANN; TREMBLAY, 1996). Tal suposição é corroborada por Houmard et al.(1996), citando que, após a cessação de um programa de exercício físico, as adaptações com relação à concentração de GLUT4 são, em grande parte, perdidas.

No que diz respeito às características do programa de exercícios físicos a serem prescritos não observa-se consenso na literatura. Em se tratando da intensidade do esforço, Braun, Zimmermann e Kretchmer (1995) concluíram que o exercício físico realizado com baixa intensidade demonstrou maior efetividade na sensibilidade à insulina que aquele realizado com alta intensidade. Imbeault et al. (1997) demonstraram que, com relação ao balanço entre a ingestão e a demanda energética, o exercício físico de alta intensidade mostrou-se mais efetivo em favorecer um balanço energético negativo (e portanto uma maior perda de gordura corporal) que aquele realizado em baixa intensidade.

Tremblay, Simoneau e Bouchard (1994) concordam no ponto relacionado à maior efetividade no balanço energético negativo para o exercício físico de alta intensidade. No entanto, segundo estes autores, ao se partir de um ponto de vista clínico, é óbvio que altas intensidades de esforço não poderão ser prescritas para indivíduos com risco de disfunções ou para indivíduos obesos que jamais fizeram uso do exercício físico. Nestes casos, o mais prudente seria esforços de baixa intensidade, com elevação progressiva na frequiência e na duração das sessões. Seria mais apropriado o exercício físico vigoroso ao invés de baixa intensidade, quando as condições físicas o permitissem. Por sua vez, Lynch et al. (1996) sugerem que a adoção de um programa de exercícios físicos realizado em intensidade moderada (considerada para estes autores como intensidade suficiente para elevar o metabolismo de repouso em valores maiores ou iguais a 5,5 METs) representará maior foco para a prevenção primária do diabetes mellitus tipo 2, patologia que apresenta grande ligação com a obesidade centrípeta.

Aparentemente não é apenas o exercício aeróbio que poderá exercer influência positiva nos níveis de adiposidade centrípeta e em suas conseqüências deletérias à saúde. Treuth et al. (1995) observaram em mulheres na pós-menopausa redução na gordura corporal da região intra-abdominal após programa de exercícios físicos envolvendo resistência de força, o qual consistiu em exercícios de musculação para membros inferiores, superiores e tronco, por 16 semanas, freqüência de 3 sessões /semana, uma hora por sessão, aumentando-se a carga progressivamente de 50\% de uma repetição máxima no início para cerca de $67 \%$ no final do programa. Neste estudo, uma diminuição na relação entre o tecido adiposo intraabdominal e o tecido adiposo abdominal subcutâneo proporcionou evidências de que o programa de exercícios de força propiciou uma utilização preferencial do tecido adiposo visceral como fonte energética.

Outrossim, Miller et al. (1994) observaram que o treinamento de força proporcionou elevação na sensibilidade à insulina, diminuindo os níveis deste hormônio em homens idosos e de meia idade, aparentemente de forma independente do consumo máximo de oxigênio, composição corporal e distribuição da gordura, havendo contudo a necessidade de estudos adicionais que focalizem as mudanças no mecanismo de transporte da glicose na musculatura esquelética nesta forma de exercício físico. 
Nota-se portanto a necessidade de pesquisas para verificar a qualidade e quantidade ideal de exercícios físicos, objetivando a prevenção da obesidade centrípeta em indivíduos geneticamente suscetíveis e a alteração da gordura central subcutânea e intraabdominal em indivíduos já acometidos, para melhora no perfil de risco induzido pelo estado obeso, evitando-se o advento das disfunções a ele associadas.

\section{Conclusão}

O presente estudo relacionado à obesidade centrípeta, salientando seu papel em distúrbios metabólicos, além da mensuração e do papel do exercício físico em sua prevenção e tratamento permite inferir que tal patologia, notadamente no que diz respeito ao compartimento intra-abdominal, apresenta extensa importância clínica, relacionando-se de forma direta com diversos distúrbios. Estes distúrbios aparentam ser decorrentes de excessiva lipogênese e lipólise nesta região, conduzindo a um excessivo transporte de ácidos graxos livres na circulação hepática (veia porta) e, como consequiência a uma diminuição no clearance de insulina hepática, hiperinsulinemia, diminuição da sensibilidade à insulina, diabetes mellitus e finalmente aterosclerose.

Uma anormalidade genética relacionada ao códon 64 no gen de receptores $b_{3}$ adrenérgicos aparenta ser uma das causas do acúmulo de gordura na região visceral, além de ser o elo de ligação entre a obesidade central e tais distúrbios, apesar de anormalidades endócrinas relacionadas aos hormônios esteróides e ao hormônio do crescimento, juntamente com hábitos inadequados de ingestão calórica e sedentarismo que aparentam estar também envolvidos.

A mensuração da deposição de gordura na região central poderá ser realizado principalmente por intermédio de métodos de imagem, sobretudo pela tomografia computadorizada e ressonância nuclear magnética, podendo contudo ser estimada pela antropometria (medidas de circunferência).
Finalmente, o exercício físico, principalmente aliado ao controle alimentar, pode exercer importante papel na reversão da gordura central, tanto subcutânea quanto visceral, a níveis metabolicamente suportáveis. Com relação ao exercício aeróbio, o aumento na quantidade e eficiência do transportador de glicose GLUT4 aparenta ser um dos motivos da melhora apresentada nos padrões somáticos e metabólicos apresentados. Apesar de exercícios de força aparentemente também exercerem papel importante, não se verificou até o presente como se dá a ação de tais atividades no metabolismo, porém o transportador de glicose GLUT4 também deve desempenhar um papel importante neste aspecto. Outrossim, com relação à prescrição dos exercícios físicos, os esforços de baixa a moderada intensidade parecem ser os mais indicados. Todavia, não aparenta estar claro qual o tipo, a duração, a frequiência e a intensidade ótimos para prevenção ou reversão do quadro apresentado.

\section{Referências}

AMERICAN COLLEGE OF SPORTS MEDICINE. Guidelines for exercise testing and prescription. 5.ed. Philadelphia, Willians \& Wilkins, 1995.

ARSLANIAN, S.; SUPRASONGSIN, C. Insulin sensitivity, lipids, and body composition in chilhood: Is "syndrome $\mathrm{X}$ ” present ? Journal of Clinical Endocrinology and Metabolism, Baltimore, v.81, p.1058-1062, 1996.

ARNER,P.. The $b_{3}$ - adrenergic receptor - A cause and cure of obesity?. New England Journal of Medicine, Waltham, v.333, n.6, p.382-383, aug.1995.

BENTHEM, L. et al. Excess portal venous long-chain fatty acids induce Syndrome $\mathrm{X}$ via HPA axis and sympathetic activation. American Journal of Physiology Endocrinol. Metabolism and Gastrointestinal Physiology, Bathesda, v.279, E1286-E1293, 2000.

BJÖRNTORP, P. Regional patterns of fat distribution. Annals of Internal Medicine, Philadelphia, v.103, p.994995, 1985.

. Portal adipose tissue as a generator of risk factors for cardiovascular disease and diabetes.Arteriosclerosis, v.10, n.4,p.493-496, 1990. 
Silva, J. L. T. da et al.

.Visceral fat accumulation: The missing link between psychosocial factors and cardiovascular disease? Journal of Internal Medicine, Oxford, v.230, p.195-201, 1991

Insulin resistance: the consequence of a neuroendocrine disturbance? International Journal of Obesit, London, v.19, suppl.1, p.S6-S10, 1995a.

Evolution of the understanding of the role of exercise in obesity and its complication. International Journal of Obesity, London, v.19, suppl. 4, p.s1-s4, 1995b.

The android woman. A risk condition. Journal of Iinternal Medicine, Oxford, v.293, p.105-110, 1996.

Endocrine abnormalities in obesity. Diabetes Reviews, Alexandria, v.5, n.1, p.52-68, 1997.

BOUCHARD,C. Genetic factors in the regulation of adipose tissue distribution. Acta Medica Scandinavica, Stockholm, Suppl.723, p.135-141, 1988.

BOUCHARD, C. et al.. The reponse to long-term overfeeding in identical twins. New England Journal of Medicine, Waltham, v.322, n.21, p. 1477-1482, 1990.

BOKYO, E.J. et al. Visceral adiposity, fasting plasma insulin, and blood pressure in japanese-americans. Diabetes Care, New York, v.18, n.2, p.174-181, 1995.

BRANCHTEIN, L. et al. Waist circumference and waistto-hip ratio are related to gestacional glucose tolerance. Diabetes Care, New York, v.20, n.4, 1997.

BRASIL. Ministério da Saúde. Manual de diabetes. 2 ed. Brasília, 1993.

BRAUN, B.; ZIMMERMANN; M.B.; KRETCHMER,N. Effects of exercise intensity on insulin sensitivity in women with non-insulin-dependent diabetes mellitus. Journal of Applied Physiology, Bethesda, v.78, n.1, p.300-306, 1995.

BUEMANN, B.; TREMBLAY, A. Effects of exercise training on abdominal obesity and related metabolic complications. Sports Medicine, Auckland, v.21, n.3, p.191-212, 1996.

BUSETTO, L. et al. Assessment of abdominal fat distribution in obese patients: Anthropometry versus computerized tomography. International Journal of Obesity, London, v.16, p.731-736, 1992.

BUSETTO, L. Visceral obesity and the metabolic syndrome: Effects of weight loss. Nutrition, Metabolism and $C$ ardiovascular Diseases, Milano, v.11, p.195-204, jun. 2001.

CAREY, D.G. et al. Abdominal fat and insulin resistance in normal and overweight women. Diabetes, New York, v.45, p.633-638, 1996.
CLASEY, J.L. et al. Body composition by DEXA in older adults: accuracy and influence of scan mode. Medicine and Science in Sport and Exercise, Baltimore, v.29, n.4, p.560-567, 1997.

CLÉMENT, K. et al. Genetic variation in the $b_{3}$-adrenergic receptor and an increased capacity to gain weight in patients with morbidy obesity. New England Journal of Medicine,Waltham, v.333, p.352-354, 1995.

COLDITZ, G.A. Economic costs of obesity. American Journal of Clinical Nutrition, New York, v.55, p.503s507s, 1992.

COLMAN, E. et al. Body fatness and waist circumference are independent predictors of the age-associated increase in fasting insulin levels in health men and women. International Journal of Obesity, London, v.19, p.798$803,1995$.

DEFRONZO, R.A.; FERRANNINI, E. Insulin resistance. A multifaceted syndrome responsible for NIDDM, obesity, hypertension, dyslipidemia, and atherosclerotic cardiovascular disease. Diabetes Care, New York, v. 14, n.13, p. 173-194, 1991.

DENGEL, D.R. et al. Distinct effects of aerobic exercise training and weight loss on glucose homeostasis in obese sedentary men. Journal of Applied Physiology, Bethesda, v.81, n.1, p.318-325, 1996.

DESPRÉS, J.P. et al. Physical training and changes in regional adipose tissue distribution. Acta Medica Scandinavica, Stocholm, suppl 723, p.205-212, 1988.

DESPRÉS, J.P. et al. Role of deep abdominal fat in the association between regional adipose tissue distribution and glucose tolerance in obese women. Diabetes, New York, v.38, p.304-309, 1989a.

Regional distribution of body fat, plasma lipoproteins and cardiovascular disease. Arteriosclerosis, Dallas, v.10, n.4, p.497-511, 1990.

Imaging techniques applied to the measurement of human body composition. In: ROCHE, A.F.; HEYMSFIELD, S.B.; LOHMAN, T.G. Human Body Composition. Champaign Human Kinetics, 1996. p.149166.

ELBEIN, S.C. et al. Role of the B3-adrenergic receptor locus in obesity and noninsulin-dependent among members of caucasian families with a diabetic sibling pair. Journal of Clinical Endocrinology and Metabolism, Baltimore, v.81, p.4422-4427, 1996.

FISBERG, M. Obesidade na infância e adolescência. In: FISBERG, M. et al. Obesidade na infância e adolescência. Fundo editorial BYC. São Paulo. P. 09-13, 1995. 
FUJIMOTO, W.Y. et al. Susceptibility to development of central adiposity among populations. Obesity Research, Boston, suppl.2, p.179s-186s, 1995.

GIACOBINO, J.P. Beta 3 - adrenoceptor: an update. European Journal of Endocrinology, Oslo, v.132, p.377$385,1995$.

HANSEN, N.J. et al. Prediction of body composition in premenopausal females from dual energy $\mathrm{x}$-ray absorptiometry. Journal of Applied Physiology, Bethesda, v.75, n.4, p.1637-1641, 1993.

HENNES, M.M; SHRAGO, E.; KISSEBAH, A.H. Receptor and postreceptor effects of free fat acids (FFA) on hepatocyte insulin dynamics. International Journal of Obesity, London, v.14, n.10, p.831-841, 1990.

HOUMARD, J.A.et al. Seven days of exercise increase GLUT-4 protein content in humans skeletal muscle. Journal of Applied Physiology, Bethesda, v.79, n.06, p.1936-1938, 1995.

HOUMARD, J. A. et al. Effect of reduced training and training cessation on insulin action and muscle GLUT4. Journal of Applied Physiology, Bethesda, v.81, n.3, p.1162$1168,1996$.

HUBERT, H.B. et al. Obesity as an independent risk factor for cardiovascular disease: A 26-year follow-up of participants in the Framingham heart study. Circulation, Baltimore, v.67, n.5, p.968-977, 1983.

HOFFSTEDT, J. et al. The metabolic syndrome is related to $b_{3}$ - adrenoceptor sensitivity in visceral adipose tissue. Diabetologia, New York, v.39, p.838-844, 1996.

HUNTER, G.R. et al. Fat distribution, physical activity, and cardiovascular risk factors. Medicine and Science in Sports and Exercise, Baltimore, v.29, n.3, p.362-369, 1997.

IMBEAULT, P. et al. Acute effects of exercise on energy intake and feeding behaviour. British Journal of Nutrition, Cambridge v.77, p.511-521, 1997.

JAKICIC, J.M. et al. Association between blood lipids and different measures of body fat distribution: effects of BMI and age. International Journal of Obesity, London, v.17, p.131-137, 1993.

JEBB, S.A. Measurement of soft tissue composition by dual energy x-ray absorptiometry. British Journal of Nutrition, Cambridge v.77, p.151-163, 1997.

JOHANNSSON, G. et al. Growth hormone treatment of abdominally obese men reduces abdominal fat mass, improves glucose and lipoprotein metabolism, and reduces diastolic blood pressure. Journal of Clinical Endocrinology and Metabolism, Baltimore, v.82, p.727734, 1997.
KALKHOFF, R.K. et al. Relationship of body fat distribution to blood pressure, carbohydrate tolerance, and plasma lipids in healthy obese women. The Journal of Laboratory and Clinical Medicine, St. Louis, v.102, p.621-627, 1983.

KAPLAN, N.M. The deadly quartet: Upper body obesity, glucose intolerance, hypertriglyceridemia and hypertension. Archives of Internal Medicine, Chicago, v.149, p.1514-1519, 1989.

KELLEY, D.E. et al. Subdivisions of subcutaneous abdominal adipose tissue and insulin resistance. American Journal of Physiology Endocrinology Metabolism and Gastroenterology Physiology, Bethesda, v.278, E941E948, 2000.

KIRWAN, J.P. et al. Regular exercise enhances activation of IRS-1-associated PI3-kinase in human skeletal muscle. Journal of Applied Physiology, Bethesda, v.88, n.2, p.797803,2000

KISSEBAH, A.H. et al. Relation of body fat distribution to metabolic complications of obesity. Journal of Clinical Endocrinology and Metabolism, Baltimore, v.54, p.254260, 1982.

KISSEBAH, A.H.; PEIRIS, A.N.; EVANS, D.J. Mechanisms associating body fat distribution to glucose intolerance and diabetes melito: window with a view. Acta Medica Scandinavica, Stockholm, suppl.723, p.79-89, 1988.

KISSEBAH, A.H. Central obesity: measurement and metabolic effects. Diabetes Reviews, v.5, n.1, p.8-20, 1997.

KLUTE, R.; SCHUBERT, A. Obesity in Europe. Annals of Internal Medicine, Philadelphia, v.103, n.6(part 2), p.10371042, 1985.

KOHRT, W.M.. Body composition by DXA: Tried and true ? Medicine and Science in Sports and Exercise, v.27, n.10, p.1349-1353, 1995.

KURIYAMA, H. et al. Enhanced expression of hepatic acyl-coenzime A synthetase and microsomal triglyceride transfer protein messenger RNAs in the obese and hypertriglyceridemic rat with visceral fat accumulation. Hepatology, Baltimore, v.27, p.557-562, 1998.

LEHMANN, R. et al. Loss of abdominal fat and improvement of the cardiovascular risk profile by regular moderate exercise training in patients with NIDDM. Diabetologia, New York, v.38, p.1313-1319, 1995.

LOHMAN, T.G. Advances in body composition assessment. Champaign, Human Kinetics 1992, p. 57-63.

LÖNNROTH, P. Potential role of adipose tissue for the development of insulin resistance in obesity. Acta Medica Scandinavica, Stockholm, suppl. 723, p.91-94, 1988. 
LOWELL, B.B; FLIER, J.S..The potential significance of $\mathrm{b}_{3}$ adrenergic receptors. Journal of Clinical Investigation, New York, v.95, p.923, 1995.

LYNCH, J. et al. Moderately intense physical activities and high levels of cardiorespiratory fitness reduce the risk of non-insulin-dependent diabetes mellitus in middleaged men. Archives of Internal Medicine, Chicago, v.156, p.1307-1314, 1996.

MACOR, C. et al. Visceral adipose tissue impairs insulin secretion and insulin sensitivity but not energy expenditure in obesity. Metabolism, v.46, n.2, p.123-129, 1997.

MARIN, P. et al. Cortisol secretion in relation to body fat distribution in obese premenopausal women. Metabolism, v.41,p.882-886, 1992.

MASUZAKI, H. et al. Transgenic Model of Visceral Obesity and the Metabolic Syndrome. Science, Boston, v.294, n.5549, p.2166-2170, 2001.

MAURIÈGE, P.; BOUCHARD, C. Trp64Arg mutation in b3-adrenoceptor gene of doubtful significance for obesity and insulin resistance. The Lancet, New York, v.348, p.698699, 1996.

MAYES, P.A. Transporte e armazenamento de lipídeos. In: MURRAY, R.K. et al. Harper: Bioquímica, 8.ed. São Paulo, Atheneu, 1998 p.254-270.

McARDLE, W.D.; KATCH, F.I.; KATCH, V.L. Essentials of Exercise Physiology, Philadelphia, Lea \& Febiger, 1994.

McKEIGUE, P.M.; MARMOT, M.G. Mortality from coronary heart disease in Asian communities in London. British Medical Journal, Edinburgh, v. 297, p.903, 1988.

MILLER, J.P. et al. Strength training increases insulin action in healthy 50-to-65-yr-old men. Journal of Applied Physiology, Bethesda, v.77, n.3, p.1122-1127, 1994.

MUST, A. et al. Long term morbidity and mortality of oveweight adolescents: a follow-up of the Harvard growth study of 1922 to 1935. The New England Journal of Medicine, Waltham, v.327, p.1350-1355, 1992.

NAGASE, T. et al. Lack of association between the $\operatorname{Trp}^{64} \mathrm{Arg}$ mutation in the B3 adrenergic receptor gene and obesity in japanese men: A longitudinal analysis. Journal of Clinical Endocrinology and Metabolism, Baltimore, v.82, p.1284-1287, 1997.

NAKAJIMA, T. et al. Correlation of intraabdominal fat accumulation and left ventricular performance in obesity. The American Journal of Cardiology, New York, v 64, p.369-373, 1989.

NATIONAL INSTITUTES OF HEALTH CONSENSUS DEVELOPMENT PANEL ON THE HEALTH IMPLICATIONS OF OBESITY. Health Implications of obesity. Annals of Internal Medicine, Philadelphia, v.103, p. 1073-1077, 1985.

NEWSHOLME, E. A.; LEECH, A.R. Biochemistry for the medical sciences. J. Wiley \& Sons, 1985.p.93-166.

PASQUALI, R. et al. The hypothalamic-pituitary-adrenal axis in obese women with different patterns of body fat distribution. Journal of Clinical Endocrinology and Metabolism, Baltimore, v.77, n.2, p.341-346, 1993.

PEIRIS, A.N. et al. Relationship of anthropometric measurements of body fat distribution to metabolic profile in premenopausal women. Acta Medica Scandinavica, Stockholm, suppl.723, p.179-188, 1988.

PICCINI, R.X.. Obesidade: constituição, atividade ou educação ? Revista da Associação Médica Brasileira, São Paulo, v.42, n.2, p.79-83, 1996.

POULIOT, M.C. et al. Waist circumference and abdominal sagittal diameter: Best simple anthropometric indexes of abdominal visceral adipose tissue accumulation and related cardiovascular risk in men and women. The American Journal of Cardiology, New York, v.73, p.460468, 1994.

REAVEN, G.M. Role of insulin resistance in human disease. Diabetes, New York, v.37, p.1595-1607, 1988.

RÖNNEMAA, T. et al. Glucose metabolism in identical twins discordant for obesity. The critical role of visceral fat. Journal of Clinical Endocrinology and Metabolism, Baltimore, v.82, n.2, p.383-387, 1997.

ROSMOND, R. BOUCHARD, C., BJÖRNTORP, P. TSP 509I polimorphism in exon 2 of the glucocorticoid receptor gene in relation to obesity and cortisol secretion: Cohort study. British Medical Journal, Edinburgh , v.322, p.652$653,2001$.

ROSS, R. Effects of diet and exercise-induced weight loss on visceral adipose tissue in men and women. Sports Medicine, Auckland, v.24, n.1, p.55-64, 1997.

SAMÁRAS, K. et al. Independent genetic factors determine the amount and distribution of fat in women after the menopause. Journal of Clinical Endocrinology and Metabolism, Baltimore, v.82, p.781-785, 1997.

SILVER, K. et al. Molecular scanning for mutations in the b3- adrenergic receptor gene in Nauruans with obesity and noninsulin dependent diabetes mellitus. Journal of Clinical Endocrinology and Metabolism, Baltimore, v.81, n.11,p.4155-4158, 1996.

SILVA, J.L.T. Quantidade/distribuição de gordura corporal, capacidade cardiorrespiratória, nível de estresse e presença de fatores componentes da síndrome metabólica. 1999, 218 p. Dissertação (Mestrado em Ciência do 
Movimento Humano) - Universidade Federal de Santa Maria, 1999.

TREMBLAY, A.; SIMONEAU, J.; BOUCHARD, C. Impact of exercise intensity on body fatness and skeletal muscle. Metabolism.v. 43, n.7, p.814-818, 1994.

TREUTH, M.S. et al. Reduction in intra-abdominal adipose tissue after strength training in older women. Journal of Applied Physiology, Bethesda, v.78, n.4, p.1425-1431, 1995.

VAGUE, J. La différenciation sexuelle. Facteur déterminant des formes de l'óbésité. Presse Medicale, Paris, v.55, p.339$340,1947$.

. The degree of masculine differentiation of obesities: A factor determining predisposition to diabetes, atherosclerosis, gout, and uric calculous disease. The American Journal of Clinical Nutrition, New York, v.4, n.1, p.20-34, 1956.

VAN ITALLIE, T.B. Health implications of overweight and obesity in the United States. Annals of Internal Medicine, Philadelphia, v.103, n.6 (part 2), p.983-988, 1985.

YAMASHITA, S. et al. Insulin resistance and body fat distribution. Diabetes Care, New York, v.19, n.3, p.287291, 1996.
YOUNG, T.K.; GELSKEY, D.E. Is noncentral obesity metabolically begnign? Journal of The American Medical Association, Schaumburg, v.274, n.24, p.1939-1941, 1995.

WALSTON, J. et al. Time of onset of non-insulindependent diabetes mellitus and genetic variation in the $\mathrm{b}_{3}$-adrenergic-receptor gene. New England Journal of Medicine, Waltham, v.333, p.343-347, 1995.

WÍDEN, E. et al. Association of a polymorphism in the $\mathrm{b}_{3}$-adrenergic-receptor gene with features of the insulin resistance syndrome in Finns. New England Journal of Medicine, Waltham, v.333, p.348-351, 1995.

WILMORE, J.H., COSTILL, D.L. Physiology of Sport and Exercise. Champaign: Human Kinetics, 1994.

WOLF, G. Glucocorticoids in adipocytes stimulate visceral obesity. Nutrition Reviews, New York, v.60, p. 148-151, 2002. 
